Rafael Fabiano Machado Rosa ${ }^{1}$

Raquel Papandreus DibI ${ }^{2}$

Nadima Viera Toscan ${ }^{3}$

LeONARDO LeirIa MOURA DA SIIIA ${ }^{3}$

Paulo Ricardo Gazzola Zen ${ }^{4}$

Carla Graziadio ${ }^{5}$

GIORGIO AdRIANO PASKULIN ${ }^{6}$

\section{Amenorréia primária e cariótipo XY: identificando pacientes em risco}

\author{
Primary amenorrbea and XY karyotype: identifying patients in risk
}

Palavras-chave

Amenorréia

Cromossomo $Y$

Diferenciação sexual

Receptores androgênicos

Testosterona

Diidrotestosterona

Disgenesia gonadal 46,XY

Esteróide 17-alfa-hidroxilase

Keywords

Amenorrhea

Y chromosome

Sexual differentiation

Androgen receptors

Testosterone

Dihydrotestosterone

$46, X Y$ gonadal dysgenesis

17 alpha-hydroxylase

\section{Resumo}

OBJETIVO: verificar a prevalência e as características clínicas de pacientes com amenorréia primária e cariótipo XY avaliadas em nosso Serviço com o intuito de identificar achados que possam auxiliar em seu reconhecimento. MÉTODOS: no período de Janeiro de 1975 a Novembro de 2007, foram avaliadas 104 pacientes com amenorréia primária. Para todos os casos foi realizada a análise pelo cariótipo por bandas GTG. Destas, 21 (20,2\%) apresentavam uma constituição 46,XY. Contudo, duas foram excluídas do estudo por terem prontuários incompletos. Das 19 pacientes que compuseram a amostra, a maior parte veio encaminhada pela ginecologia $(63,2 \%)$. Suas idades variaram entre 16 e 41 anos (média de 22, 1 anos). Realizou-se uma coleta de dados sobre sua história familiar e pregressa, exame físico e resultados de exames complementares. Para determinação dos seus diagnósticos levaram-se em consideração essas informações. RESULTADOS: a síndrome de resistência aos androgênios foi o diagnóstico predominante $(n=12 ; 63,2 \%)$. Cinco pacientes $(26,3 \%)$ apresentavam disgenesia gonadal pura XY (DGP XY), uma (5,3\%) deficiência de 17-alfa hidroxilase e uma $(5,3 \%)$ deficiência de 5-alfa redutase. Achados clínicos freqüentemente observados nessas pacientes incluíram desenvolvimento anormal dos caracteres sexuais secundários $(n=19)$, agenesia uterina com vagina em fundo de saco $(n=14)$, história familiar de amenorréia (n=8) e gônadas palpáveis no canal inguinal (n=5). Duas delas apresentavam história de hérnia inguinal. Hipertensão arterial sistêmica foi diagnosticada somente na paciente com deficiência de 17-alfa hidroxilase, e malignização gonadal, naquela com DGP XY. CONCLUSÕES: a freqüência de pacientes com cariótipo XY (20\%) foi superior à usualmente descrita na literatura (3 a 1 1\%). Acreditamos que isso tenha relação com a forma de encaminhamento das pacientes ao Serviço. Certos achados da história clínica e do exame físico deveriam ser rotineiramente avaliados em indivíduos com amenorréia primária. Dessa forma, haveria um reconhecimento mais precoce das pacientes 46,XY e, conseqüentemente, um adequado manejo clínico das mesmas.

\section{Abstract}

PURPOSE: to verify the prevalence and clinical characteristics of patients with primary amenorrhea and XY caryotype, evaluated in our Service, aiming at identifying findings which could help their recognition. METHODS: from January 1975 to November 2007, 104 patients with amenorrhea were evaluated. All the cases were analyzed by the caryotype by GTG bands. Among them, 21 (20.2\%) presented a XY 46 constitution. Nevertheless, two of them were excluded from the study, because of incomplete data in their patient's chart. Most of the 19 patients who formed the sample had been referred to us by the gynecology clinics (63.2\%). Their ages varied from 16 to 41 years old (an average of 22. 1). Data were collected about their family and previous history, physical examination and results of complementary exams and the information was taken into consideration to determine the diagnosis. RESULTS: the predominant diagnosis was resistance to androgens syndrome $(n=12 ; 63.2 \%)$; five patients (25.3\%) presented XY pure gonadal dysgenesis (XY PGD), one
Correspondência:

Giorgio Adriano Paskulin

Genética Clínica - UFCSPA

Avenida Sarmento Leite, 245, sala 403 - Centro CEP 90050-170 - Porto Alegre/RS Fone: (51) 3303-8771/Fax: (51) 3303-8810 E-mail: paskulin@ufcspa.edu.br

Recebido

23/9/08

Aceito com modificacões

$17 / 11 / 08$
Universidade Federal de Ciências da Saúde de Porto Alegre - UFCSPA - Porto Alegre (RS), Brasil; Complexo Hospitalar Santa Casa de Porto Alegre - CHSCPA - Porto Alegre (RS), Brasil.

Geneticista Clínico da Universidade Federal de Ciências da Saúde de Porto Alegre - UFCSPA - Porto Alegre (RS), Brasil; e Complexo Hospitalar Santa Casa de Porto Alegre - CHSCPA - Porto Alegre (RS), Brasil; Pós-graduando (Doutorado) do Programa de Pósgraduação em Patologia da UFCSPA - Porto Alegre (RS), Brasil

2 Preceptora do Serviço de Ginecologia e Obstetrícia do Complexo Hospitalar Santa Casa de Porto Alegre - CHSCPA - Porto Alegre (RS), Brasil; Pós-graduanda (Doutorado) do Programa de Pós-graduação em Patologia da Universidade Federal de Ciências da Saúde de Porto Alegre - UFCSPA - Porto Alegre (RS), Brasil.

${ }^{3}$ Acadêmicos do Curso de Medicina da Universidade Federal de Ciências da Saúde de Porto Alegre - UFCSPA - Porto Alegre (RS), Brasi. ${ }^{4}$ Geneticista Clínico da Universidade Federal de Ciências da Saúde de Porto Alegre - UFCSPA - Porto Alegre (RS), Brasil; e Complexo Hospitalar Santa Casa de Porto Alegre - CHSCPA - Porto Alegre (RS), Brasil; Professor Adjunto da Disciplina de Genética Clínica da UFCSPA - Porto Alegre (RS), Brasil.

${ }^{5}$ Geneticista Clínica da Universidade Federal de Ciências da Saúde de Porto Alegre - UFCSPA - Porto Alegre (RS), Brasil; e Complexo Hospitalar Santa Casa de Porto Alegre - CHSCPA - Porto Alegre (RS), Brasil; Professora-assistente da Disciplina de Genética Clínica da UFCSPA - Porto Alegre (RS), Brasil.

- Geneticista Clínico da Universidade Federal de Ciências da Saúde de Porto Alegre - UFCSPA - Porto Alegre (RS), Brasil; e Complexo Hospitalar Santa Casa de Porto Alegre - CHSCPA - Porto Alegre (RS), Brasil; Citogeneticista Responsável pelo Laboratório de Citogenética da UFCSPA - Porto Alegre (RS), Brasil; Professor-associado da Disciplina de Genética Clínica da UFCSPA - Porto Alegre (RS), Brasil. 
(5.3\%) 17 alpha-hydroxylase deficiency, and one (5.3\%), 5 alpha-reductase deficiency. Clinical findings frequently found in these patients included abnormal development of secondary sexual characters $(n=19)$, uterine agenesia with a blind vagina $(n=14)$, family history of amenorrhea $(n=8)$, and palpable gonads in the inguinal canal $(n=5)$. Two of them presented a history of inguinal hernia. Systemic arterial hypertension was only diagnosed in the patient with 17 alphahydroxylase deficiency, and gonadal malignization, in the one with XY PGD. CONCLUSIONS: the rate of patients with XY caryotype (20\%) was higher than the one described in the literature (3 to $11 \%$ ). It is believed that this fact is related to the way patients are usually referred to our service. Some findings from the clinical history and from the physical examination should be evaluated as a routine in individuals with primary amenorrhea. This way, there would be a more precocious detection of XY 46 patients, and a better clinical management of them, as a consequence.

\section{Introdução}

A amenorréia representa um sintoma, e não uma doença, sendo que a ausência de menstruação caracteriza a amenorréia do tipo primária. Essa é uma condição com diferentes causas, como disfunções endocrinológicas e anomalias gonadais, sendo que dentre elas destacam-se as anormalidades cromossômicas ${ }^{1,2}$. Essas últimas começaram a ser identificadas a partir da década de 1950, com o desenvolvimento das técnicas de cultivo celular associadas à utilização da colchicina e de soluções hipotônicas para o tratamento das metáfases, o que possibilitou uma melhor avaliação dos cromossomos ${ }^{3}$. Atualmente, sabe-se que de 16 a $63 \%$ das pacientes com amenorréia primária são portadoras de uma anormalidade cromossômica, sendo que entre 3 e $18 \%$ apresentam uma alteração genética associada a uma constituição cromossômica $46, \mathrm{XY}^{1,4,5}$.

A importância de essas pacientes serem reconhecidas reside principalmente no fato de que, pela presença de um cromossomo $\mathrm{Y}$, elas apresentam um risco aumentado de malignização gonadal ${ }^{1-3,5-7}$. Além disso, esses indivíduos possuem anormalidades somáticas e funcionais decorrentes do distúrbio da diferenciação sexual, como agenesia uterina e gônadas disgenéticas, com grandes repercussões sobre sua fertilidade. Muitas necessitam ainda de terapia precoce com estrogênios para indução da puberdade e melhoria da densidade óssea (prevenção da osteoporose). Apesar de sua freqüência e da importância do seu reconhecimento, são poucos os estudos com descrições clínicas dessas pacientes em nosso meio ${ }^{6,8}$. A familiarização dos profissionais da área de saúde, em especial pediatras, endocrinologistas e ginecologistas, com as características clínicas apresentadas por essas pacientes é essencial para identificação e manejo clínico adequados.

Assim, o objetivo deste trabalho foi verificar a prevalência e as características clínicas de pacientes com amenorréia primária e cariótipo XY diagnosticadas em nosso Serviço, com o intuito de identificar achados que possam auxiliar no seu reconhecimento precoce.

\section{Métodos}

Foram incluídas em nosso estudo, inicialmente, todas as pacientes encaminhadas por amenorréia ao Serviço de Genética Clínica da Universidade Federal de Ciências da Saúde de Porto Alegre (UFCSPA), no período de janeiro de 1975 a novembro de 2007. O total foi de 147 pacientes, sendo que, dentre elas, $104(70,7 \%)$ apresentavam amenorréia do tipo primária. Em todas foi realizada com sucesso a avaliação cromossômica através do cariótipo com bandamento GTG (banda G por tripsina e giemsa), com uma contagem média de 25 placas metafásicas por paciente. Resumidamente, essa técnica inclui a preparação de cultivos celulares a partir de sangue periférico seguida de tratamento com colchicina, choque hipotônico, fixação das células e preparação das lâminas para posterior coloração e análise ao microscópio.

Foram incluídas no trabalho somente as pacientes que apresentavam cariótipo 46,XY. Das 104 pacientes com amenorréia primária, $21(20,2 \%)$ possuíam uma constituição cromossômica XY. Contudo, duas delas foram excluídas do estudo por terem prontuários médicos com descrição clínica incompleta. A amostra final foi constituída, portanto, por 19 pacientes. Suas idades, no momento da primeira avaliação, variaram de 16 a 41 anos (a média foi de 22,1 anos, sendo que 36,8\% apresentavam idade superior a 20 anos). A maior parte foi encaminhada pelos serviços de ginecologia $(63,2 \%)$ e endocrinologia $(26,3 \%)$, sendo que seis delas $(31,6 \%)$ já apresentavam suspeita clínica de constituição cromossômica XY no momento do encaminhamento.

Através de uma revisão sistemática de suas fichas de atendimento, realizou-se uma análise retrospectiva das características clínicas, laboratoriais e radiológicas dessas 19 pacientes. Foram anotados dados referentes à história familiar, tanto de amenorréia como de consangüinidade entre os pais, história de desenvolvimento de tumores nas gônadas e de realização de gonadectomia profilática, além de idade da pubarca e telarca (quando presentes). Ao exame físico, foram verificados a altura, presença e desenvolvimento dos caracteres sexuais secundários (pêlos axilares e pubianos, mamas), clitoromegalia, gônadas palpáveis, hérnia inguinal e vagina em fundo de saco. Foram anotados, também, os resultados das dosagens hormonais de estradiol, LH (hormônio luteinizante), FSH (hormônio folículo-estimulante), testosterona total (T) e dihidrotestosterona (DHT), bem como da avaliação ultra-sonográfica abdominal e pélvica e do anatomopatológico das gônadas retiradas. A altura das pacientes foi avaliada a partir de curvas de crescimento padrão para indivíduos adultos do sexo feminino, e os caracteres sexuais secundários através de tabelas com os estágios de Tanner. 
Para a determinação dos diagnósticos apresentados pelas pacientes foram levados em consideração todos os dados obtidos dos prontuários. O diagnóstico de síndrome de resistência aos androgênios (SRA) foi realizado com base nos achados clínicos apresentados pelas pacientes (em especial, agenesia uterina, desenvolvimento de mamas com falta/ escassez de pêlos axilares e pubianos e presença de gônadas palpáveis) somado a dosagens hormonais aumentadas de $T$ para o padrão feminino (sem alteração na relação T/DHT) . No caso da disgenesia gonadal pura XY (DGP XY), o mesmo foi determinado pela falta de desenvolvimento de caracteres sexuais secundários e presença de sistema Mülleriano ${ }^{10,11}$. O diagnóstico da deficiência de 5-alfa redutase baseou-se, principalmente, no achado de aumento acentuado da relação T/DHT basal, às custas de uma redução da DHT e elevação da testosterona total em decorrência da falta de conversão periférica da mesma, associado à presença de gônadas palpáveis e próstata e ausência de desenvolvimento das mamas ${ }^{12}$. A deficiência de 17-alfa hidroxilase foi determinada a partir de dosagens baixas de testosterona e estradiol, e altas de LH e FSH, juntamente com agenesia uterina, hipertensão arterial sistêmica (HAS) e hipocalemia ${ }^{13}$.

O processamento e a análise dos dados foram realizados com o uso dos programas Microsoft ${ }^{\circledR}$ Excel 2002 e PEPI (versão 4.0). Para comparação da freqüência de pacientes com cariótipo XY observada em nossa amostra em relação aquela descrita nos demais trabalhos da literatura, foi utilizado o teste exato de Fisher bicaudado, considerando-se significantes valores de $\mathrm{p}<0,05$.
Este estudo foi aprovado pelo Comitê de Ética em Pesquisa da Universidade Federal de Ciências da Saúde de Porto Alegre (UFCSPA).

\section{Resultados}

O diagnóstico de SRA foi o predominante, observado em 12 pacientes $(63,2 \%)$. Cinco $(26,3 \%)$ apresentavam DGP XY, uma $(5,3 \%)$ deficiência de 17 -alfa hidroxilase e uma $(5,3 \%)$ deficiência de 5 -alfa redutase (Tabela 1$)$. História familiar de amenorréia foi verificada em oito pacientes $(42,1 \%)$, sete delas $(87,5 \%)$ com SRA. A outra foi a paciente com deficiência de 17-alfa hidroxilase. Em três casos, foi detectada consangüinidade: duas portadoras de SRA e uma com deficiência de 5-alfa redutase (Tabela 1 e Figura 1).

Quanto ao desenvolvimento dos caracteres sexuais secundários, observou-se que as pacientes com SRA apresentaram telarca, com desenvolvimento de mamas em estágio de Tanner IV-V, ausência de pêlos axilares e pêlos pubianos escassos (Tanner I-II). Uma delas possuía uma hipertrofia leve de clitóris. Por sua vez, pacientes com DGP XY desenvolveram mamas e pêlos pubianos somente após o início da terapia com estrogênios. Duas delas apresentavam, ainda, uma hipertrofia leve do clitóris. As pacientes com deficiência de 17-alfa hidroxilase e deficiência de 5-alfa redutase não possuíam desenvolvimento mamário, e os pêlos pubianos e axilares eram presentes, porém, escassos. Gônadas palpáveis no canal inguinal foram descritas em cinco pacientes, quatro

Tabela 1 - Diagnósticos e características clínicas apresentadas pelas 19 pacientes de nossa amostra

\begin{tabular}{|c|c|c|c|c|c|}
\hline Diagnóstico & SRA & DGP XY & $\begin{array}{c}\text { Deficiência de } \\
\text { 17-alfa hidroxilase }\end{array}$ & $\begin{array}{l}\text { Deficiência de } \\
\text { 5-alfa redufase }\end{array}$ & Total \\
\hline Média (anos) & 22,7 & 21,2 & 23 & 19 & - \\
\hline Ginecologia & 9 & 2 & - & 1 & 12 \\
\hline Endocrinologia & 2 & 2 & 1 & - & 5 \\
\hline \multicolumn{6}{|l|}{ Achados clínicos } \\
\hline Hipertrofia de clitóris & 1 & 2 & - & - & 3 \\
\hline Gônadas palpáveis & 4 & - & - & $\cdot$ & 4 \\
\hline Hérnia inguinal & 2 & - & - & - & 2 \\
\hline Agenesia uterina & 12 & - & 1 & 1 & 14 \\
\hline Tumor de células germinativas & - & 1 & - & - & 1 \\
\hline Consangüinidade & 2 & - & - & 1 & 3 \\
\hline História familiar de amenorréia & 7 & - & 1 & - & 8 \\
\hline Total (\%) & $12(64)$ & $5(26)$ & $1(5)$ & $1(5)$ & $19(100)$ \\
\hline
\end{tabular}

$S R A=$ síndrome de resistência aos androgênios; DGP $X Y=$ disgenesia gonadal pura $X Y$; HAS= hipertensão arterial sistêmica; Tumor= história de malignização gonadal. 
delas com SRA e uma com deficiência de 5-alfa redutase. Duas pacientes $(10,6 \%)$ apresentavam história de hérnia inguinal, ambas com SRA. Agenesia uterina com vagina em fundo de saco foi observada em 13 pacientes $(68,4 \%)$, o que corresponde àquelas com SRA $(\mathrm{n}=11)$, deficiência de 17-alfa hidroxilase $(n=1)$ e deficiência de 5-alfa redutase $(n=1)$. A paciente com deficiência de 5 -alfa redutase apresentava, ainda, uma próstata reduzida de volume, mas palpável ao toque retal. HAS foi diagnosticada somente na paciente com deficiência de 17-alfa hidroxilase. A altura das pacientes com SRA variou de 153 a $175 \mathrm{~cm}$ (média de $164 \mathrm{~cm}$; percentil $75 \rightarrow 50$ ) e a das mulheres com DGP XY oscilou de 153 a $172 \mathrm{~cm}$ (média de $162 \mathrm{~cm}$; percentil $25 \rightarrow 50$ ). A paciente com deficiência de 17-alfa hidroxilase apresentava $173 \mathrm{~cm}$ (percentil $90 \rightarrow 95$ ) e aquela com 5 -alfa redutase, $160 \mathrm{~cm}$ (percentil $50 \rightarrow 25$ ).

Somente uma paciente $(5,3 \%)$, com diagnóstico de DGP XY, havia apresentado malignização gonadal (desenvolvimento de disgerminoma). Em outras sete pacientes $(36,8 \%)$ havia relato de realização de cirurgia de gonadectomia profilática após o diagnóstico do distúrbio do desenvolvimento sexual XY. Em nenhuma delas, contudo, houve detecção de células tumorais nas gônadas retiradas. A Tabela 1 sumariza os principais

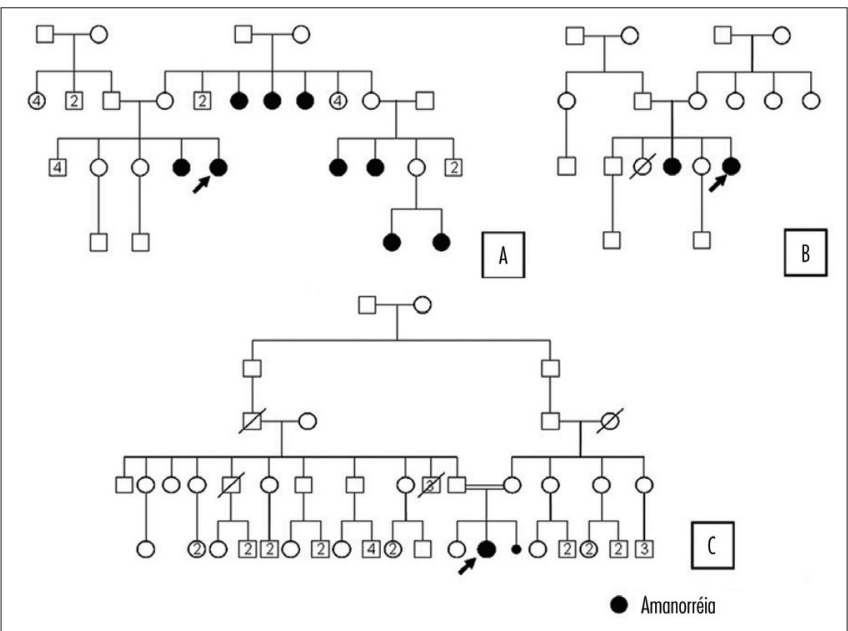

Figura 1 - Heredogramas das famílias de três pacientes de nossa amostra As setas indicam os respectivos casos (probandas). A primeira (A) é de uma das pacientes com a síndrome de resistência aos androgênios, apresentando uma história familiar de amenorréia bastante rica. A doença é passada ao longo das gerações através das respectivas mães, que são normais fenotipicamente, mas portadoras da mutação para a síndrome. Essas não são acometidas, pois apresentam uma constituição cromossômica 46 XX. $\bigcirc$ segundo (B) corresponde ao da família da paciente com deficiência de 17-alfa hidroxilase. Note que ela apresenta também uma irmã afetada (só que, como vimos antes, com uma constituição cromossômica $46 \mathrm{XX}$ ), sendo que esse padrão de acometimento (linear, em uma só geração) é bastante sugestivo de uma condição autossômica recessiva, como é o caso da doença em questão. $\bigcirc$ terceiro heredograma $(\mathrm{C})$ é da paciente com diagnóstico de deficiência de 5-alfa redutase. Note a consangüinidade presente entre seus pais, relacionada com a etiologia da doença. diagnósticos e achados clínicos apresentados pelas pacientes de nossa amostra.

\section{Discussão}

Distúrbios da produção ou ação dos androgênios podem resultar em virilização ausente ou mesmo deficiente, tanto dos genitais externos como nos internos, caracterizando o antigamente chamado pseudo-hermafroditismo masculino, hoje definido como distúrbio do desenvolvimento sexual XY ${ }^{14,15}$. Apesar de esses indivíduos apresentarem, freqüentemente, anormalidades somáticas decorrentes desse desenvolvimento genital anormal já ao nascimento, muitos acabam sendo diagnosticados somente tardiamente, na adolescência e idade adulta, devido a alterações menstruais e falta de desenvolvimento dos caracteres sexuais secundários. Nesta amostra de pacientes com amenorréia primária, verificou-se que um número significativo delas $(36,8 \%)$ foi diagnosticado apenas tardiamente, após os 20 anos. O cariótipo convencional, a partir de sangue periférico (leucócitos), é usualmente suficiente para sua identificação. Contudo, a avaliação das características clínicas dessas pacientes é freqüentemente necessária para a determinação da doença genética específica associada à constituição cromossômica XY. Dessa forma, a familiarização de profissionais da área de saúde com esses achados é fundamental para o reconhecimento precoce desses indivíduos.

A freqüência de pacientes com amenorréia primária e cariótipo XY encontrada em nosso estudo (20\%) foi estatisticamente similar à de trabalhos descritos na literatura que relataram índices entre 14 e $18 \%{ }^{1}$. Diferenças significativas foram encontradas em relação aos estudos que descreveram valores que oscilaram de 3 a $11 \%$ 1,3,16,17. Acreditamos que essas diferenças tenham associação com as formas de seleção e metodologia empregadas nos trabalhos. Na maioria das investigações citogenéticas de mulheres com amenorréia primária as pacientes têm sido freqüentemente selecionadas pela natureza de suas anomalias somáticas ${ }^{4}$. Acredita-se que, em nosso caso, o alto índice de pacientes com cariótipo XY tenha relação com o fato de nossa amostra ter sido constituída principalmente de indivíduos referenciados por suspeita de uma alteração cromossômica.

As duas doenças genéticas com constituição cromossômica XY mais freqüentes entre pacientes com amenorréia primária, tal como observado em nosso estudo, são a SRA e a DGP XY. As deficiências de 17alfa hidroxilase e 5-alfa redutase são raras. Entretanto, em todos os casos é importante entender que a falência ovariana não é diretamente causada pelos cromossomos, uma vez que essas pacientes apresentam uma constituição 
cromossômica normal do sexo masculino. Ela resulta de uma alteração a nível gênico que leva a um distúrbio do desenvolvimento sexual ${ }^{1}$.

A SRA, diagnóstico mais freqüente de nossa amostra, de acordo com a literatura, é uma forma bastante rara de distúrbio do desenvolvimento sexual, com uma incidência estimada de um caso para $20 \mathrm{mil} \mathrm{a} 99 \mathrm{mil}$ homens. Conhecida também como síndrome de Morris, ela é considerada, por outro lado, uma das causas mais freqüentes de distúrbio do desenvolvimento sexual XY, sendo observada em cerca de 5 a $9 \%$ das pacientes com amenorréia primária ${ }^{2,9,18,19}$ (em nosso estudo essa freqüência foi de 12\%). Essa é uma doença recessiva ligada ao $\mathrm{X}$ na qual indivíduos $46, \mathrm{XY}$ apresentam um déficit parcial ou completo da ação do androgênio devido a anormalidades no seu receptor. O gene codificador do mesmo localiza-se na região 11-12 do braço longo do cromossomo $\mathrm{X}^{9,18-21}$, sendo que o padrão de herança apresentado pela SRA pode ser bem visualizado pelo heredograma de uma das pacientes de nossa amostra, exposto na Figura 1A. A história de consangüinidade verificada em dois casos não se relaciona com a etiologia da doença.

Indivíduos com a forma completa da SRA, tal como as pacientes de nossa amostra, apresentam-se, mais comumente, com um fenótipo feminino acompanhado, na idade adulta, de amenorréia primária ${ }^{18,20}$. Essas pacientes se caracterizam, tal como observado em nosso estudo, por um quadro clínico com desenvolvimento de mamas, mas ausência ou escassez de pêlos axilares e pubianos, e agenesia uterina com vagina curta em fundo cego. Essa última ocorre devido à presença dos testículos e, conseqüentemente, produção do fator inibidor Mülleriano (FIM) que, como o próprio nome diz, inibe o desenvolvimento dos ductos de Müller (útero e terço proximal da vagina). Os testículos podem se mostrar palpáveis no canal inguinal, como verificado em quatro pacientes deste trabalho $(33,3 \%)$, sendo que, algumas vezes, é acompanhado de hérnias inguinais. Esse último achado foi observado em duas pacientes $(14,3 \%)$ e poderia levar à suspeita clínica da SRA ainda durante a infância. Apesar da falta de virilização, algumas pacientes podem apresentar certo grau de fusão labioescrotal e hipertrofia de clitóris, tal como verificado em um dos casos de nossa amostra, caracterizando uma forma parcial de SRA. Como também observado em nossas pacientes, a altura média dos indivíduos com a SRA é superior àquela de mulheres normais, mas similar quando comparada a homens 46, XY $Y^{1,9,14,20-22}$.

A SRA deve ser diferenciada da agenesia Mülleriana (síndrome de Rokitansky-Küster-Hauser), pois a vagina está ausente ou encurtada em ambas. Uma forma simples de distingui-las é através da medida sérica de testosterona, que se encontra elevada em pacientes com a SRA, sendo que o diagnóstico diferencial pode ser definitivamente feito através do exame de cariótipo (o mesmo é $46 \mathrm{XX}$ em pacientes com a síndrome de Rokitansky-Küster-Hauser) $)^{2}$.

O risco de tumores de células germinativas em pacientes com a SRA é considerado inferior a $5 \%$ e ocorre, principalmente, em uma idade mais tardia, após os 25 anos de idade ${ }^{2,19}$. Em nossa amostra, nenhuma das pacientes havia apresentado malignização gonadal. $\mathrm{O}$ momento para a extirpação das gônadas ainda permanece controverso. Tradicionalmente, os testículos são deixados em seu lugar até o desenvolvimento espontâneo dos caracteres sexuais secundários. Por outro lado, outros investigadores recomendam que se realize a orquiectomia tão cedo o diagnóstico seja feito, pois carcinomas testiculares in situ têm sido diagnosticados mesmo em crianças, e tumores invasivos de células germinativas, em pacientes púberes ${ }^{19}$.

A DGP XY, por sua vez, é uma condição geneticamente heterogênea observada em aproximadamente $5 \%$ das pacientes com amenorréia primária ${ }^{1,2}$ (em nosso estudo, a freqüência foi similar). Conhecida também por síndrome de Swyer, essa é uma anormalidade resultante da regressão embrionária dos testículos induzida, algumas vezes (cerca de $20 \%$ dos casos), por mutações no gene SRY $Y^{10,11,23,24}$. Ela pode ser tanto herdada na forma ligada ao X como autossomicamente ${ }^{1}$. Caracteriza-se clinicamente, como observado pelas pacientes de nosso estudo, por um fenótipo feminino com amenorréia primária, infantilismo sexual e desenvolvimento das estruturas Müllerianas devido à falta de produção do FIM (os testículos nessas pacientes são disgenéticos) ${ }^{10,11,14,23}$. As pacientes não apresentam anormalidades somáticas associadas à síndrome de Turner, como a baixa estatura; ao contrário, possuem freqüentemente uma estatura normal-alta ou mesmo alta para o padrão feminino ${ }^{3}$. O desenvolvimento dos caracteres sexuais secundários ocorre, usualmente, após o início da hormonioterapia ${ }^{10,11}$, como verificado nas pacientes de nosso estudo.

Essa aparência dos indivíduos com a DGP XY pode ser clinicamente idêntica a aparência daqueles com DGP $\mathrm{XX}$. Em ambas as condições, as gonadotrofinas (FSH e LH) mostram-se, após a puberdade, elevadas e os estrogênios diminuídos, sendo a diferenciação feita usualmente pelo exame de cariótipo. Algumas pacientes com DGP XY podem apresentar uma hipertrofia leve de clitóris, como verificado em dois indivíduos de nossa amostra, sendo que esse achado pode auxiliar na diferenciação clínica entre ambas as condições ${ }^{2,3}$.

Ao contrário de indivíduos com a SRA, as gônadas de pacientes com a DGP XY não secretam hormônios e deveriam ser sempre removidas no momento do 
diagnóstico. Disgerminomas ou gonadoblastomas desenvolvem-se em cerca de 20 a $30 \%$ das pacientes com DGP XY, especialmente durante a primeira ou segunda década de vida ${ }^{2,10,11,23}$. A incidência desses tumores aumenta com a idade, ocorrendo de 50 a $70 \%$ aos 30 anos e de $80 \%$ aos $40 \operatorname{anos}^{23}$. De forma interessante, a única paciente de nossa amostra com malignização gonadal, uma paciente com DGP XY, apresentava gônadas em sua posição habitual na avaliação ultra-sonográfica. Contudo, verificou-se posteriormente que ambas já representavam massas tumorais (disgerminomas).

Por outro lado, a síntese de esteróides sexuais requer que as vias de biossíntese adrenal e gonadal estejam intactas. Hiperplasia adrenal congênita é o termo utilizado para um grupo de distúrbios genéticos causado por defeitos em qualquer uma das cinco vias enzimáticas que levam à síntese do cortisol a partir do colesterol.

A deficiência de 17-alfa hidroxilase é uma anormalidade autossômica recessiva bastante rara. $\mathrm{O}$ gene CYP 17, que codifica a enzima, está localizado no braço longo do cromossomo 10, na região 24-25, e é expresso nas supra-renais e nas gônadas ${ }^{13}$. A deficiência dessa enzima resulta em uma redução da síntese de cortisol com conseqüente aumento da secreção de ACTH e mineralocorticóides, além da falta de produção tanto de androgênios como de estrogênios. Em indivíduos com constituição cromossômica 46,XY, a deficiência da 17-alfa hidroxilase pode levar a um quadro de hipogonadismo hipergonadotrófico com amenorréia, ausência de desenvolvimento de mamas e pêlos axilares e pubianos e uma genitália feminina com vagina em fundo cego, como observado em nossa paciente. Esse último achado se deve à produção do FIM pelo testículo, que pode estar localizado na região abdominal, inguinal ou labioescrotal ${ }^{13,25,26}$. A HAS com hipocalemia ocorre em decorrência do excesso de produção de mineralocorticóides. No caso de nossa paciente, o diagnóstico foi feito aos 12 anos de idade. Curiosamente, como observado no heredograma da Figura 1B, ela possuía uma irmã também portadora de deficiência de 17-alfa hidroxilase, com amenorréia primária e HAS, só que com uma constituição cromossômica 46 XX e, conseqüentemente, presença das estruturas Müllerianas. Testículos localizados na região intra-abdominal ou inguinal devem também ser removidos cirurgicamente devido à alta freqüência de tumorigênese ${ }^{13}$.

Embora estudos preliminares postulassem que a DHT fosse um metabólito inativo da $\mathrm{T}$, a descoberta de uma doença rara da diferenciação sexual, causada pela deficiência da enzima 5 -alfa redutase 2 , mostrou o contrário ${ }^{8}$. Inicialmente denominada hipospadia perineoescrotal pseudovaginal, a deficiência de 5-alfa redutase é uma doença genética autossômica recessiva. O gene codificador desta enzima, o SRD5A2, localizase no braço curto do cromossomo 2 na região $23^{27}$. O heredograma da Figura 1C, da paciente com deficiência de 5-alfa redutase, demonstra presença de consangüinidade entre os pais, achado este que se relaciona com a etiologia da doença. Embora tanto homens quanto mulheres possam ser homozigotos para mutações nesse gene, a expressão clínica da doença é limitada ao sexo masculino. Contudo, a variabilidade fenotípica observada nesses indivíduos é tão grande que eles podem apresentar desde um fenótipo quase masculino normal com criptorquidia até uma genitália quase feminina com presença de gônadas palpáveis ${ }^{12,27,28}$, como foi o caso de nosso paciente. Quando não orquiectomizadas, as pacientes podem apresentar sinais de virilização durante a puberdade ${ }^{12}$. Contudo, tal como observado em nossa paciente, não costumam apresentar desenvolvimento das mamas ${ }^{12}$, o que as diferencia, por exemplo, de indivíduos com a SRA.

O Quadro 1 expõe dados importantes da história clínica e do exame físico que deveriam ser rotineiramente avaliados em pacientes com amenorréia primária. Destacam-se, entre eles, a presença de agenesia uterina com vagina em fundo de saco, hérnia inguinal, hipertrofia de clitóris, gônadas palpáveis e história de tumores de células germinativas e familiar de amenorréia. Esses dados podem alertar médicos e outros profissionais da área da saúde quanto à possibilidade de se tratar uma paciente com constituição cromossômica XY. Acredita-se que essa familiarização possa colaborar com um reconhecimento mais precoce dessas pacientes e, conseqüentemente, uma adequada tomada de decisões quanto ao seu tratamento e acompanhamento clínico.

Quadro 1 - Achados importantes para avaliação de pacientes com amenorréia primária que podem auxiliar na suspeita de uma possível constituição cromossômica 46,XY

Achados clínicos
História clínica e exame físico
Estatura normal-alta ou alta
Ausência de desenvolvimento de mamas, e pêlos pubianos e axilares
Presença de mamas com ausência/ escassez de pêlos axilares e pubianos
Hipertrofia de clitóris
Hérnia inguinal
Gônadas palpáveis no canal inguinal ou junto aos grandes lábios
Vagina em fundo de saco
Toque retal c om palpação de próstata
Hipertensão arterial sistêmica
Radiológicos
Agenesia uterina
Tumores de células germinativas
História familiar
História familiar de amenorréia
Consangüinidade




\section{Referências}

1. Ten SK, Chin YM, Noor PJ, Hassan K. Cytogenetic studies in women with primary amenorrhea. Singapore Med J. 1990;31 (4):355-9.

2. Practice Committee of the American Society for Reproductive Medicine. Current evaluation of amenorrhea. Fertil Steril. 2006;86/5 Suppl):S148-55.

3. van Niekerk WA. Chromosomes and the gynecologist. Am J Obstet Gynecol. 1978;130(8):862-75.

4. Opitz $\mathrm{O}$, Zoll B, Hansmann I, Hinney B. Cytogenetic investigation of 103 patients with primary or secondary amenorrhea. Hum Genet. 1983;65(1):46-7.

5. Wong MS, Lam ST. Cytogenetic analysis of patients with primary and secondary amenorrhoea in Hong Kong: retrospective study. Hong Kong Med J. 2005; 11 (4):267-72.

6. D'Agostini C, Gus R, Capp E, Corleta HVE. Estudo citogenético das gônadas em pacientes com amenorréia primária. Rev Bras Ginecol Obstet. 2005;27(3): 125-9.

7. MacMahon RA, Cussen L, Walters WA. Importance of early diagnosis and gonadectomy in 46, XY females. J Pediatr Surg. 1980;15(5):642-5.

8. Hackel C, Oliveira LEC, Toralles MB, Nunes-Silva D, Tonini MMO, Ferraz LFC, et al. Deficiência de $5 \alpha$-redutase tipo 2: experiências de Campinas (SP) e Salvador (BA). Arq Bras Endocrinol Metab. 2005;49(1):103-11.

9. Galani A, Kitsiou-Tzeli S, Sofokleous C, Kanavakis E, KalpiniMavrou A. Androgen insensitivity syndrome: clinical features and molecular defects. Hormones (Athens). 2008;7(3):217-29.

10. Behtash N, Karimi Zarchi M. Dysgerminoma in three patients with Swyer syndrome. World J Surg Oncol. 2007;5:71.

11. Michala L, Goswami D, Creighton SM, Conway GS. Swyer syndrome: presentation and outcomes. BJOG. 2008;115(6): 737-41.

12. Hackel C, Oliveira LE, Ferraz LF, Tonini MM, Silva DN, Toralles $M B$, et al. New mutations, hotspots, and founder effects in Brazilian patients with steroid $5 \alpha$-reductase deficiency type 2 . J Mol Med. 2005;83(7):569-76.

13. Silva EA, Siqueira AC, Pacheco S, Ribeiro AK. Hiperplasia adrenal congênita-deficiência de 17 $\alpha$-hidroxilase. Arq Bras Endocrinol Metab. 2000;44(2):175-7.

14. Garel L. Abnormal sex differentiation: who, how and when to image. Pediatr Radiol. 2008;38 Suppl 3:S508-1 1.

15. Hughes IA. Disorders of sex development: a new definition and classification. Best Pract Res Clin Endocrinol Metab. 2008;22(1): 119-34.
16. Anglani F, Baccichetti C, Artifoni L, Lenzini E, Tenconi R. Frequency of abnormal karyotypes in relation to the ascertainment method in females referred for suspected sex chromosome abnormality. Clin Genet. 1984;25(3):242-7.

17. Chryssikopoulos A, Grigoriou 0 . The etiology in 77 primary amenorrhea patients. Int J Fertil. 1987;32(3):245-9.

18. Corrêa RV, Wey JC, Billerbeck AEC, Melo KFS, Mendonça BB, Wey $M V$, et al. Insensibilidade completa aos andrógenos em pacientes brasileiras causada pela mutação P766A no gene do receptor androgênico. Arq Bras Endocrinol Metab. 2005;49(1):98-102.

19. Chen $C P$, Chern SR, Wang TY, Wang W, Wang KL, Jeng CJ. Androgen receptor gene mutations in $46, X Y$ females with germ cell tumours. Hum Reprod. 1999; 14(3):664-70.

20. Melo KF, Mendonça BB, Billerbeck AE, Costa EM, Inácio M, Silva FA, et al. Clinical, hormonal, behavioral, and genetic characteristics of androgen insensitivity syndrome in a Brazilian cohort: five novel mutations in the androgen receptor gene. J Clin Endocrinol Metab. 2003;88(7):3241-50.

21. Alvarez-Nava F, Gonzalez S, Soto M, Martinez C, Prieto M. Complete androgen insensitivity syndrome: clinical and anatomopathological findings in 23 patients. Genet Couns. 1997;8(1):7-12.

22. Cheikhelard A, Morel Y, Thibaud E, Lortat-Jacob S, Jaubert F, Polak $M$, et al. Long-term follow up and comparison between genotype and phenotype in 29 cases of complete androgen insensitivity syndrome. J Urol. 2008;180(4):1496-501.

23. Iliopoulos D, Volakakis N, Tsiga A, Rousso I, Voyiatzis N. Description and molecular analysis of SRY and AR genes in a patient with 46, XY pure gonadal dysgenesis (Swyer syndrome). Ann Genet. 2004;47(2): 185-90.

24. Selvaraj K, Ganesh V, Selvaraj P. Successful pregnancy in a patient with a 46, XY karyotype. Fertil Steril. 2002;78(2):419-20.

25. D'Alberton A, Reschini E, Motta T, Catania A. Male pseudohermaphroditism due to 17-hydroxylase deficiency. J Endocrinol Invest. 1989;12(3):193-6.

26. De Marinis L, Mancini A, Saporosi A, Calabrò F, Massari M, Moneta $\mathrm{E}$, et al. Male pseudohermaphroditism caused by 17 alpha-hydroxylase deficiency. Personal case reports and a review of the literature. Minerva Ginecol. 1989;41(7):337-42.

27. Bertelloni S, Scaramuzzo RT, Parrini D, Baldinotti, Tumini S, Ghirri P. Early diagnosis of $5 \alpha$-reductase deficiency in newborns. Sex Dev. 2007; 1 (3): 147-51.

28. Baldinotti F, Majore S, Fogli A, Marrocco G, Ghirri P, Vuerich M, et al. Molecular characterization of 6 unrelated Italian patients with 5 $\alpha$-reductase type 2 deficiency. J Androl. 2008;29(1):20-8. 